\title{
Post Cholecystectomy Syndrome in a Patient due to Multiple Biliary Pathologies.
}

\author{
Bikash Bikram Thapa', Kunda Bikram Shah ${ }^{1}$, Sushil Bahadur Rawal', Srijan Malla1. \\ ${ }^{1}$ Department of Surgery, Shree Birendra Hospital.
}

\begin{abstract}
Postcholecystectomy syndrome is the recurrence of symptoms after cholecystectomy. Postcholecystectomy syndrome due to a combination of cystic duct stump calculus and choledochal cyst with recurrent choledocholithiasis is a rare presentation. This is a case report of a patient who had developed recurrent common bile duct calculi despite endoscopic removal and eventully managed with Rou-en-Y Hepaticojejunostomy for having choledochal cyst 39 years postcholecstectomy.
\end{abstract}

Keywords: choledocholithiasis, cystic duct stump calculus, choledochal cyst, postcholecystectomy syndrome.

\section{INTRODUCTION}

Postcholecystectomy syndrome (PCS) is defined as the recurrence of symptoms similar to those experienced prior to cholecystectomy. This usually takes the form of upper abdominal pain (mainly right upper quadrant) and dyspepsia, with or without jaundice ${ }^{1}$. The incidence of PCS varies from 10 to $40 \%$ and present any time between 2 days to 25 years. The recurrent common bile duct calculus is the most common cause of the post cholecystectomy syndrome. ${ }^{2}$

We report a case of of 64 years female patient who was first operated for cholelithiasis 39 years back and had undergone repeated surgical procedure for biliary pathologies. Amongst the pathologies she had suffered,postcholecystectomy syndrome associated with cystic duct stump calculus and choledochal cyst is a rare clinical condition.

\section{CASE REPORT}

A 64 year old female presented with one and half years history of right upper quadrant pain of biliary colic. On examination she was mildly icteric. She had undergone open cholecystectomy and exploration of common bile duct when she was 25 years old. 15 years later she was diagnosed with recurrent CBD stone by ERCP and had undergone sphincterotomy and stone removal. She underwent a repeat ERCP 2 years later twice for stone removal. She was operated for Meckel'sdiveticulitis when she was 57 years old.

This time her preoperative investigation revealed mildly elevated conjugated bilirubin of $1.9 \mathrm{mg} \%$ (normal< $0.4 \mathrm{mg}$ $\%)$ and elevated serum alkaline phosphatase of $318 \mathrm{U} / \mathrm{L}$ (normal $<279 \mathrm{U} / \mathrm{L}$ ). Her prothrombin time and INR were within normal limits. The ultrasound of abdomen showed dilatation of common bile duct (CBD) with multiple calculi and postcholecystectomy status. MRCP revealed choledochal cyst $(3.5 \times 3 \mathrm{~cm})$ with multiple calculi in it and dilatation of cystic duct stump(Figure 1a).

Surgery revealed type I choledochal cyst with multiple calculi, dilated cystic duct stump with a solitary calculus and a posterior sectoral duct opening directly into the choledochal cyst wall (Figure 1b). The choledochal cyst was excised, stone removed and Rou-en- $Y$ hepaticojejunostomy was performed with separate anastomosis between the posterior sectoral duct and the

\section{Correspondence:}

Maj. Dr. Bikash Bikram Thapa

Department of Surgery, Shree Birendra Hospital

Kathmandu, Nepal

Email: bbthapa@gmail.com

Phone: +977-9841276641 
jejunum (Figure 1c). She had an uneventful post operative recovery. Histological findings confirmed the choledochal cyst.

Figure 1.

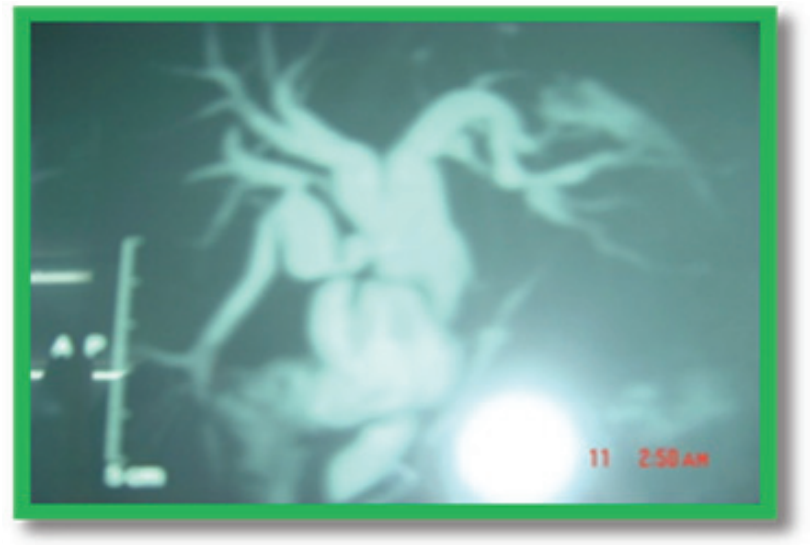

a. Cholangiography

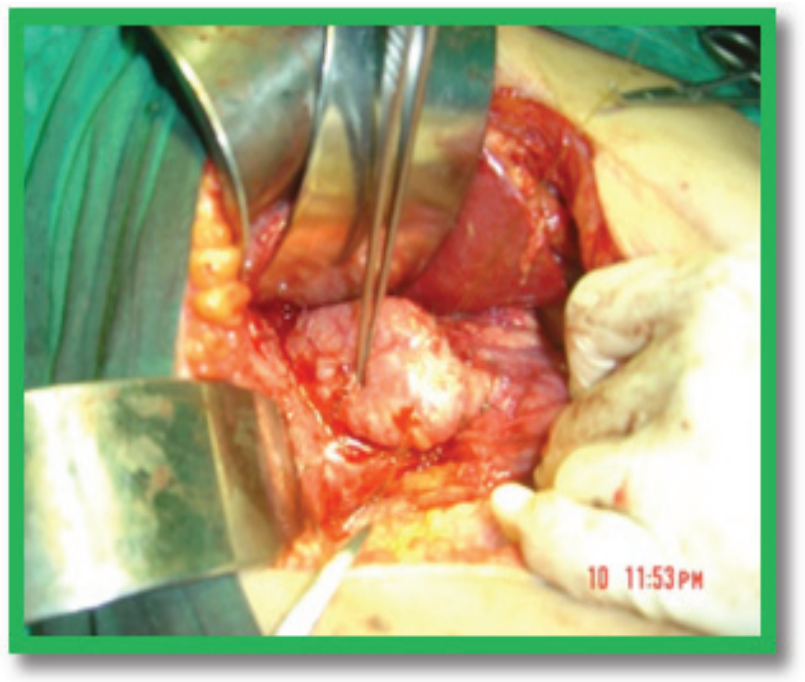

b. Choledochal cyst

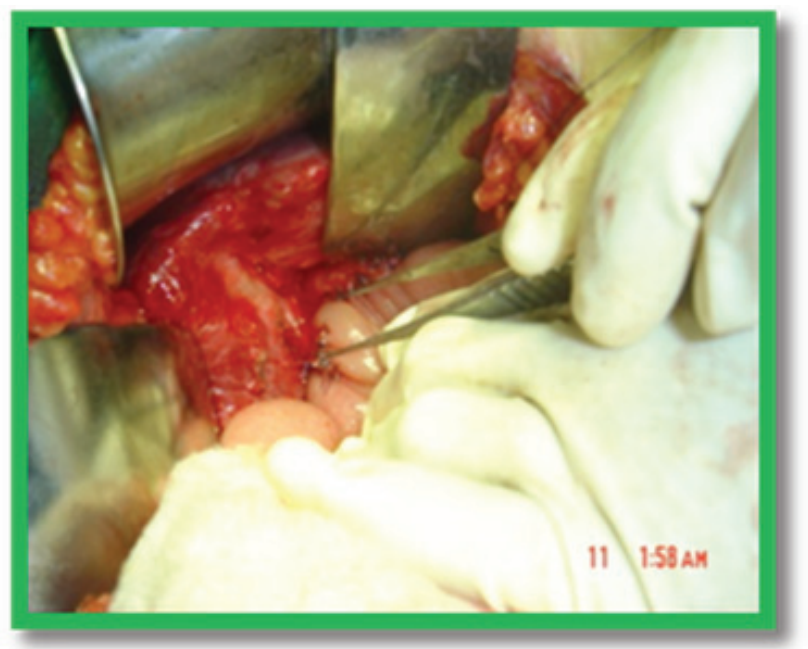

c. Stone removed

\section{DISCUSSION AND REVIEW OF LITERATURE}

Cholecystectomy, the preferred treatment for symptomatic gall bladder disease, is acknowledged to be a safe operation with low morbidity. In 1947, Womack and Crider first described PCS, defining it as the presence of symptoms after cholecystectomy. It encompasses heterogeneous group of both biliary and non biliary disorders. The incidence of postcholecystectomy syndrome has been reported to be $10-40 \%$ and the onset of symptoms may range from 2 days to 25 years with mean of 10 years ${ }^{1}$. The common biliary causes of postcholecystectomy syndrome are retained or recurrent stones, bile duct injury, bile leakage, bile duct stricture, cystic duct remnant harboring stones and inflammation, papillary stones, biliary dyskinesia etc ${ }^{2}$.

Recurrent CBD calculus is the most common cause of postcholecystectomy syndrome. In a study done by Greber et al., the incidence of retained/recurrent calculi ranged from $1.2 \%$ to $14 \%$ with only approximately $0.3 \%$ ever causing symptoms ${ }^{1}$. Patients treated endoscopically have higher incidence of retained stones compared with those treated by open exploration $10 \mathrm{Vs} 4.3 \%^{3}$. A study done by Sugiyama and colleagues showed the rate of recurrent stone formation after ERCP to be as high as $31 \%$ and demonstrated three risk factors for recurrent choledocholithiasis after endoscopic biliary sphincterotomy (EBS): (a) Common bile duct diameter $>15 \mathrm{~mm}$. (b) Periampullary diverticulum (c) Interval between initial and second EBS $<5$ years ${ }^{4}$. Though the repeated endoscopic stone extraction by ERCP when required is a safe procedure, it provides only temporary relief.

Cystic duct remnant harboring stone with or without inflammation is an uncommon cause of post cholecystectomy syndrome. Several reports have proposed that a cystic duct remnant $>1 \mathrm{~cm}$ in length after cholecystectomy may be responsible for post cholecystectomy syndrome- "cystic duct stump syndrome" ${ }^{15-8}$. The remnant is thought to cause symptoms by harboring residual calculi, serving as a site for new stone formation or by continuing to have chronic inflammation and granuloma formation ${ }^{8}$. The estimated incidence of a retained stone within the cystic duct remnant is $<2.5 \%$. Pain in the right upper quadrant was found to be the most common symptom and jaundice the commonest sign. Laparoscopic and open cholecystectomy has equal risk of cystic duct remnant calculi. Laparotomy and excision of the remnant is the treatment of choice and provides complete relief. Laparoscopic resection of the remnant, though difficult, has been performed successfully ${ }^{8}$.

Adult choledochal cyst is a rare disease condition. 
Type I Choledochal cyst manifesting for the first time in late adulthood within one year of an uneventful cholecystectomy for cholelithiasis has been reported by Mittal et al in $1993 A^{9}$. Similarly in a retrospective study, choledochal cyst has been recorded as one of the late choledochal pathology after cholecystectomy for cholelithiasis. ${ }^{2}$ Choledochal cyst typically exhibits poor drainage, even after ERCP, and is a hot bed for recurrent CBD stones and cholangitis.

Filip et al developed an algorithmic approach using endoscopic ultrasound (EUS) (Figure 2) for the initial evaluation of the patients with postcholecystectomy problems and showed the decreased number of ERCP's by $51 \%$, thus decreasing morbidity and mortality in this group of patients. However because of its capability to provide non-invasive high quality visualization of the biliary tract, magnetic resonance cholangiopancreaticography (MRCP) has been advocated as a reliable tool for identifying biliary causes of Postcholecystectomy syndrome. Accurate assessment of the site and type of biliary abnormality provides a road-map for interventional procedures, and is crucial for tailoring patients' therapy ${ }^{3}$.

Figure2.Filip et al algorithmic approach

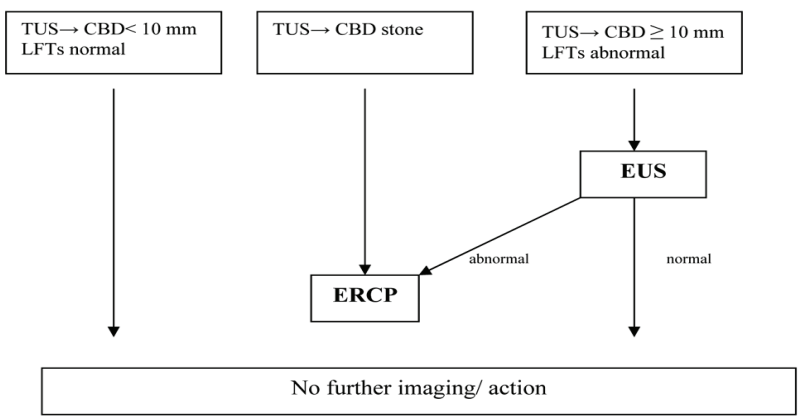

\section{CONCLUSION}

Choledochal cyst presenting in old age can be the cause of postcholecystectomy syndrome along with other biliary pathologies. Thorough preoperative evaluation and plan for definitive surgical procedure is crucial to alleviate patients' agony. The old surgical dictum "a chance to cut is a chance to cure" holds true for such multiple, rare and recurrent pathologies.

\section{REFERENCES}

1. Jaunoo SS, Mohandas S, Almond LM. Postcholecystectomy Syndrome (PCS). International journal of surgery. 2010;8:15-7.

2. Bratucu E, Straja D, Marincas M, et al. late choledochal pathology after cholecystectomy for cholelithiasis. Chirurgia (Bucur).2006;101 (3):289-95.

3. Girometti R, Brondani G, Cereser L, et al.Postcholecystectomy syndrome; Spectrum of biliary findings at magnetic resonance cholangiopancreatography. $\mathrm{Br} J$ Radiol. 2010; 83:351-61.

4. Kohn GP, Hassen AS, Banting SW, Mackay S, Cade RJ.Endoscpoic management of recurrent primary bile duct stones. ANZ J. Surg. 2008;78: 579-82.

5. Sugiyama $M$, Suzuki $\mathrm{Y}$, Masaki $T$, Mori $T$, Atomi Y. Endoscopic retreatment of recurrent choledocholithiasis after sphincterotomy. Gut. 2004;53:1856-9.

6. Walsh RM, Ponsky JL, Dumot J. Retained gallbladder /Cystic duct remnant calculi as a cause of postchlecystectomy pain. SurgEndosc. 2002; 16:981-4.

7. Shaw C, O'Hanlon DM, Fenlon HM, McEntee GP. Cystic duct remnant and the 'post-cholecystectomy syndrome.' Hepatogastoenterology. 2004;51:36-8.

8. Federick LV, Sukriya N, Benjamin P, Nihar RP. Gallbladder Remnant and cystic duct stump calculus as a cause of postcholecystectomy syndrome. Tropical Gastroenterology. 2005;26:159-60.

9. ErangaP, Shweta B, Vikram SD. Cystic Duct Remnant Syndrome. Journal of clinical Imaging Science. 2011;1:2.

10. Mittal BR, Ibarullah MD, Sexaena R, Gambhir S, Das BK. Post cholecystectomy syndrome due to choledochal cyst: detection by biliary scintigraphy. Indian Journal of Nuclear Medicine.1993;8:45-6.

11. Monalisa F, Adrian S, Carmen P, ET al. Postcholecystectomy Syndrome- an Algorithmic Approach. J Gastrointestin Liver Dis. 2008; 18 (1):67-71. 